\title{
ORAL REHABILITATION WITH OSSEO INTEGRATED IMPLANTS WITHIN THE NATIVE BONE FOLLOWING BENIGN TUMOR OR CYSTIC LESION ABLATION
}

\author{
Ahmed S. Naguib* and Rafik R. Beder**
}

\begin{abstract}
Purpose: This study aimed to determine the prognosis of performing oral rehabilitation with osseo-integrated implants within the native bone, following tumor or cystic ablation.

Patients \& Methods: This study was conducted on eight adult patients, (3) males and (5) females, their ages ranged from 25-45 years in Oral and Maxillofacial Surgery Department. Faculty of Dentistry, Tanta University. Stage (I) followed up using panoramic radiograph immediately and then after 6 months. stage (II), The inserted fixtures followed up both clinically and radiographically immediately, three and six months. stage (III), prosthetic rehabilitation. All patients followed up clinically and radiographically, after four months.
\end{abstract}

Results: No pain had detected around the inserted fixtures in all cases, except in case no. (3). No peri- implant infection occurred in all cases except in case no. (3). No implant mobility had been detected in all cases, except in case no. (3). Peri-implant radiolucency had been detected around the fixtures of case no. (3) only, and proper osseo-integration occurred in all other cases. orthopantogramic and $\mathrm{CBCT}$ views showed absence of bone resorption around the inserted fixtures in all cases, except in case no. (3). Excellent patient's satisfaction occurred in all patients regarding to facial contour, but according to aesthetic and functional aspects, only one patient (case no.3) was unsatisfied.

Conclusion: Installation of dental fixtures in the native bone of the lower Jaw after cystic or tumor ablation considered as an excellent Modality for performing oral rehabilitation

KEY WORDS: VAS:Visual analogue scale. CBCT: Cone beam computerized tomogram.

\footnotetext{
* Ass. Professor, Oral and Maxillofacial Surgery Dept., Faculty of Dentistry, Zagazig University.
}

** Ass. Professor, Oral and Maxillofacial Surgery Dept., Faculty of Dentistry, Tanta University. 


\section{INTRODUCTION}

The process of oral rehabilitation for patients who suffered from mandibular defects following marginal resection of the tumors is challenging up till now. ${ }^{(1)}$

The process of marginal mandibular defect may lead to occurrence of deformity in the facial contour and impairment in oral function such as mastication and swallowing difficulty, pooling and drooling of saliva, speech affection with associated psychological problems. ${ }^{(2)}$

The restoration of function after tumor ablation from the oral cavity, constitutes one of the big challenges that facing most of the head and neck oncologists. (3) It is known properly that, the unfavorable anatomy of the intraoral soft and hard tissues that occurs after tumors ablation often constitutes an insurmountable problem for dental rehabilitation. ${ }^{(4)}$

The properly Osseo integrated dental implants offered major advances in the oral rehabilitation of patients after ablation of oral neoplasms, as they provide proper stabilization for the constructed prothesis. So, in this manner, patients can be rehabilitated properly by securing proper esthetic and proper function. ${ }^{(5)}$

The use of Osseo integrated implants in patients with maxillofacial defects becomes a common practice for the past 15 years. However, few studies had been reported about the results. ${ }^{(6)}$

\section{AIM OF THE STUDY}

The aim of this study was to evaluate the clinical outcome of dental implants inserted in native bone following tumor or cystic ablation.

\section{MATERIALS \& METHODS}

Eight adult patients, their ages ranged from 25 - 45 years with benign mandibular tumors or odontogenic cyst involving the teeth bearing area were included in this study. All patients received, managed and treated in the department of oral and maxillofacial surgery, Faculty of dentistry, Tanta University.

All the selected patients were entailed properly about the steps of surgical procedure including the prognosis, potential hazards and even suspected complications. They gave their approval to participate in a written informed consent. All the enrolled patients within this study (table 1) treated by marginal resection or cyst enculation followed by dental rehabilitation (after 6 months) for the lost dentition with dental implant supported fixed prothesis. All the clinical and anagraphic data for all the operated patients were reported in the following table, table (No.1)

TABLE (1): Clinical data for all the operated patients

\begin{tabular}{|c|l|c|c|c|c|}
\hline Patient No & \multicolumn{1}{|c|}{ Sex } & Age & Type of the excised tumor & No. of the inserted implants & Implant site \\
\hline 1 & Female & 25 & Cystic ameloblastoma & 5 & $34-35-36-37-38$ \\
\hline 2 & Male & 40 & Cystic ameloblastoma & 3 & $31-32-34$ \\
\hline 3 & Male & 34 & Cystic ameloblastoma & 4 & $32-33-34-41$ \\
\hline 4 & Female & 38 & Odontogenic keratocyst & 6 & $41-42-43-44-31-32$ \\
\hline 5 & Female & 31 & Cystic ameloblastoma & 3 & $34-35-37$ \\
\hline 6 & Female & 45 & Odontogenic keratocyst & 4 & $33-34-35-36$ \\
\hline 7 & Male & 42 & Ossifying fibroma & 3 & $41-42-43$ \\
\hline 8 & Female & 27 & Cystic ameloblastoma & 4 & $42-43-44-45$ \\
\hline
\end{tabular}




\section{Inclusion criteria}

1) Cooperative patient for performing periodic follow-up.

2) Have good oral hygiene with absence of any periodontal disease in the remaining teeth.

3) Absence of tumor recurrence signs and symptoms.

4) Patients seeking dental implant treatment.

5) Patients with mandibular tumors treated without segmental resection. (Fig. 1)

\section{Surgical technique for tumor or cystic ablation (Stage I surgery)}

All operations were performed under general anesthesia with nasotracheal intubation. All surgeries were performed by the same surgeon with the same operating team. Under strict aseptic conditions, all the eight patients under went marginal resection or cystic enculation. All respective procedures were performed through an intra oral approach.

\section{Postoperative care}

All patients maintained on a liquid then soft diet for 5 weeks. All patients were advised to rinse their mouth 3 times daily with $0.1 \%$ chlorohexidine solution through all the follow up periods. Also, amoxicillin clavalanic acid 1 gram was administered 2 times per day orally for two weeks postoperatively. Diclofenac potassium 50mg tablet was administered 3 times per day for two weeks postoperatively.

\section{Postoperative follow up}

A panoramic radiograph was taken at the following periods: immediate and 6 months after surgery, to check occurrences of proper bone formation and absence of any residual or recurrent lesion. (Fig. 2 \& 3)

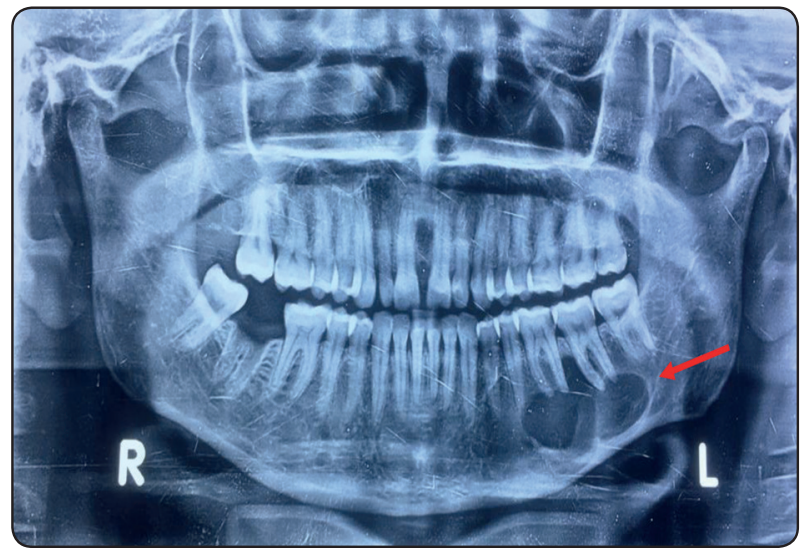

Fig. (1): Preoperative panoramic photoradiograph showing left mandibular multicystic ameloblastoma, (case no. 5)

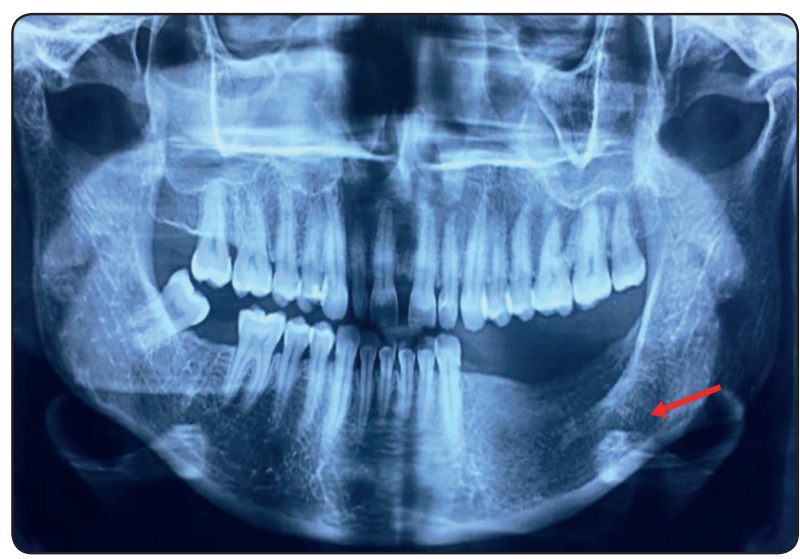

Fig. (2): Immediate postoperative panoramic photoradiograph showing complete local excision of the lesion and related teeth, (case no. 5)

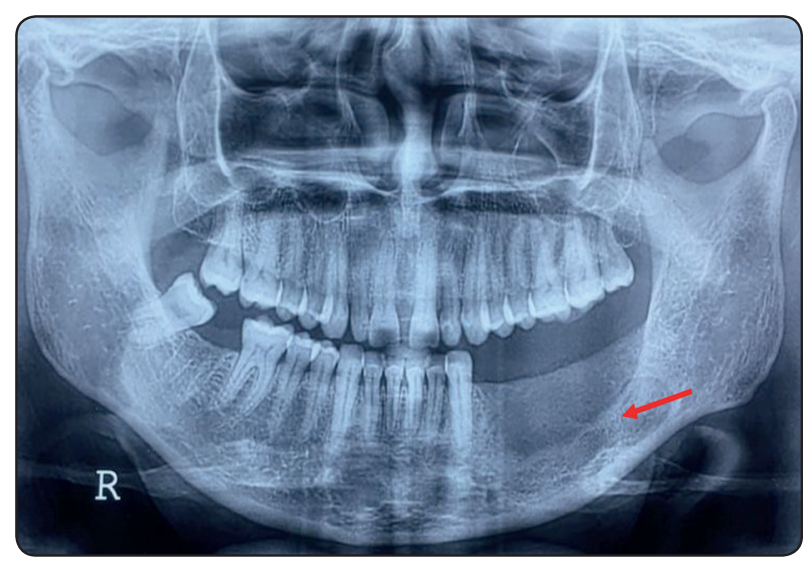

Fig. (3): Six months' postoperative panoramic photoradiograph showing lesion free bone regeneration, (case no. 5) 


\section{Surgical technique for insertion of dental implants (Stage II surgery):}

This step was performed after 6 months from performing the first surgery. Rubber base impression was obtained for each patient to construct a plaster model. Then it was sent for dental lab for construction of surgical template for each case to facilitate insertion of the dental implants in its proper place CBCT was performed to ensure the optimum volume of the regenerated bone and the proper length and width of the selected implant. (Fig. 4) A total of 32 titanium, screw- shaped oral implants (impla)* were inserted in the reconstructed areas in all 8 patients according to virtual surgical planning. (Fig.5)

\section{Postoperative follow up:}

It was performed both clinically and radiographically at the following intervals: immediately after insertion of dental implants, three months and finally six months after insertion of dental fixtures.

I- Clinical evaluation was performed by evaluating the following:

1) Absence of any persistent pain around the inserted dental implants (from the VAS) Ecklet et al $2006^{(7)}$
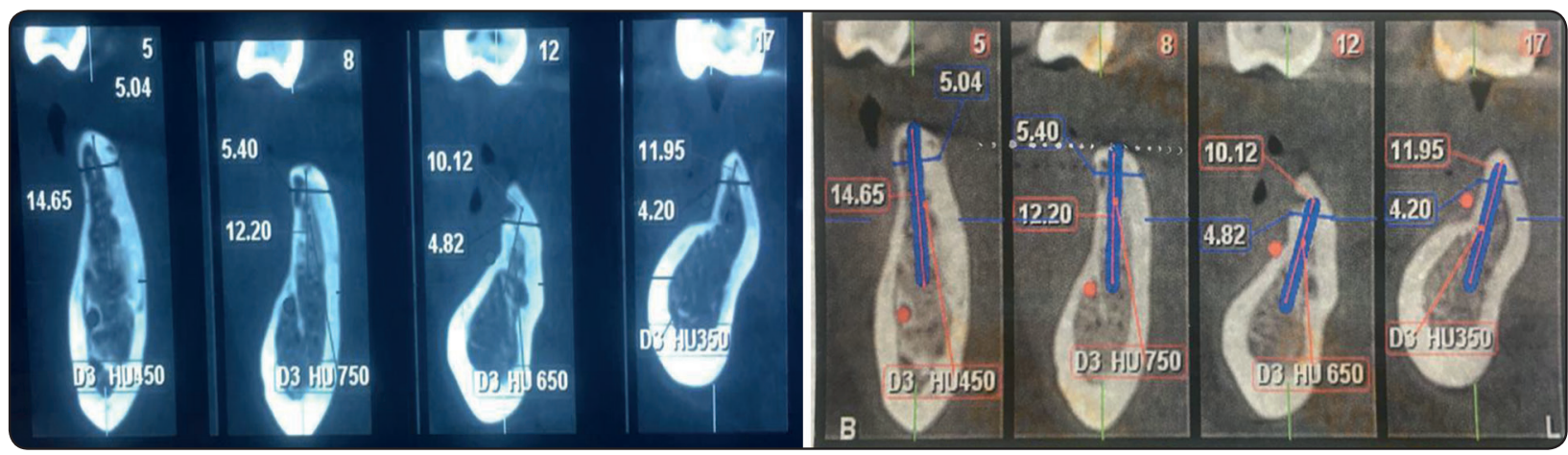

Fig. (4): Cone beam CT measuring the width, length and bone density of the regenerated ridge, (case no. 5)

* Schutz Dental GmbH. Dieselstr.5-6.D61191 Rosbach /Germany.Tel: +49(0)6003814397, Fax: +49(0)6003814902. www.schuetz-dental.com.
2) Absence of peri implant infection nor suppuration

3) Testing the implants mobility: by using OSSTELL device as follow: (Fig.6)

Stability test using OSSTELL (implant stability quotient):

- Smart peg was inserted inside implant fixture and firmly screwed to it.

- The probe of OSSTELL was directed towards the smart peg without touching it (3mm away from it) in two direction bucco-lingual and mesio-distal.

- The average of two readings was calculated.

- Values less than 60(ISQ) refers to low stability and have higher risk of failure. An increase in ISQ value during long term examination indicated that the implant more stable and good prognosis.

- ISQ value between 60 and 70 refers to medium stability.

- ISQ values more than 70 refers to high stability.

- ISQ reading was measured of time of fixture insertion as baseline, then compared with the reading at the time of final abutment insertion (6months). Valaderma etal. $2017^{(8)}$ 


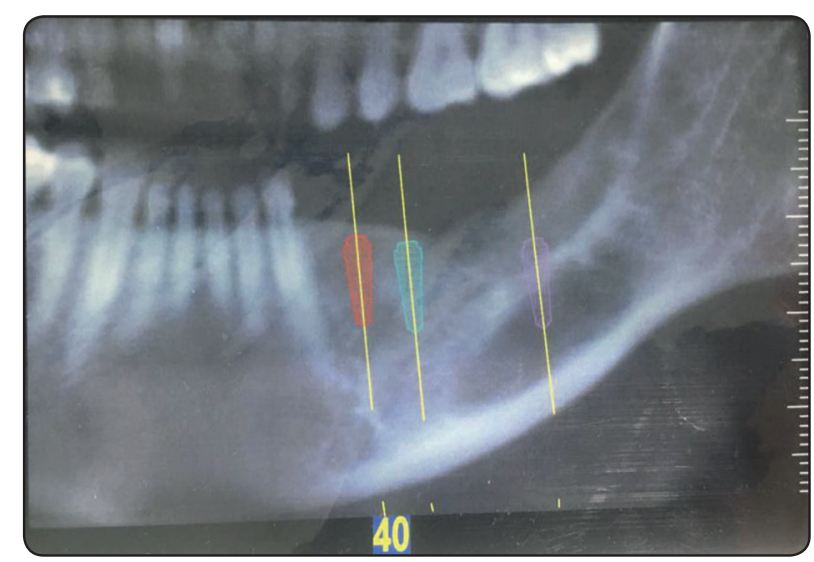

Fig. (5): Reformatted panoramic view showing virtual surgical planning and accurate implant selection, (case no. 5)

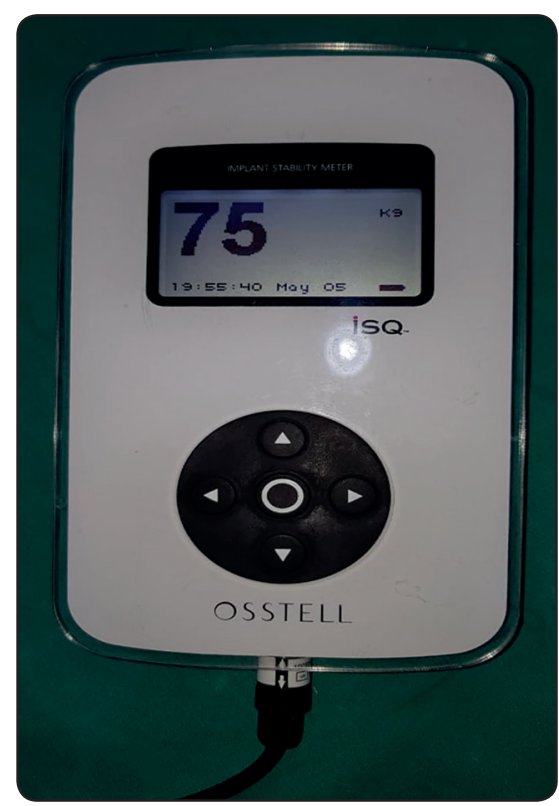

Fig. (6): Photograph showing osstell device while measuring primary implant stability

\section{Radiographic evaluation was performed by orthopantogramic and $\mathrm{CBCT}$ views to detect the following:}

1. Absence of continuous peri-implant radiolucency.

2. The degree of bone resorption around the inserted fixtures.

Measurements of changes in the level of bone height were evaluated mesial and distal to each fixture, measuring the distance from the top of fixture head shoulder to the most coronal level of direct bone to fixture contact.
TABLE (2): Mean of ISQ reading immediately and 6 months after fixture installation

\begin{tabular}{|c|c|c|}
\hline Case no. & Mean ISQ (baseline) & Mean ISQ (6 months) \\
\hline 1 & 53 & 62 \\
\hline 2 & 61 & 71 \\
\hline 3 & 59 & 50 \\
\hline 4 & 63 & 70 \\
\hline 5 & 61 & 69 \\
\hline 6 & 65 & 72 \\
\hline 7 & 66 & 73 \\
\hline 8 & 62 & 71 \\
\hline
\end{tabular}

Prosthetic rehabilitation: (stage III):

Prosthetic abutments were installated in all cases after six months from insertion of the dental implants. (Fig.7) All cases were rehabilitated with porcelain fused to metal (PFM)implants supported fixed protheses. (Fig.8)

\section{Post prosthetic rehabilitation follow up:}

It was performed after four months from prosthetic rehabilitation both clinically and radiographically as follow:

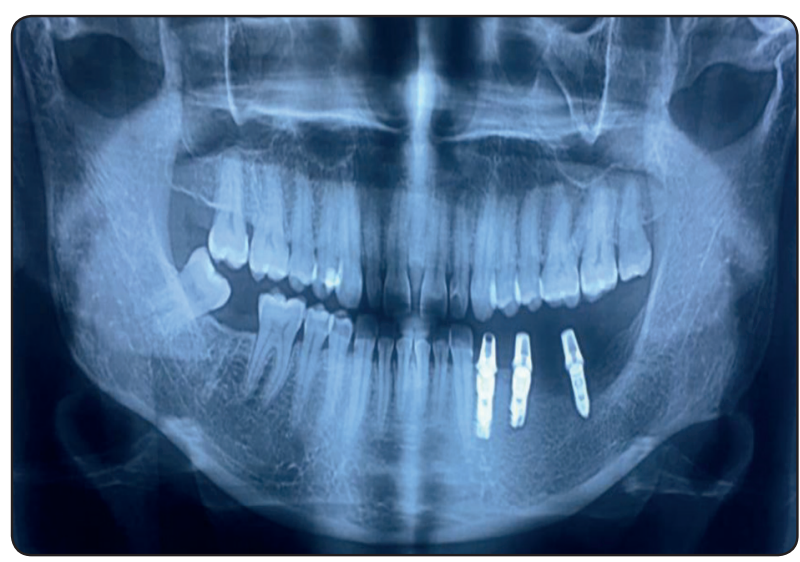

Fig. (7): Panoramic photoradiograph showing second stage installation of the selected prepared abutment 


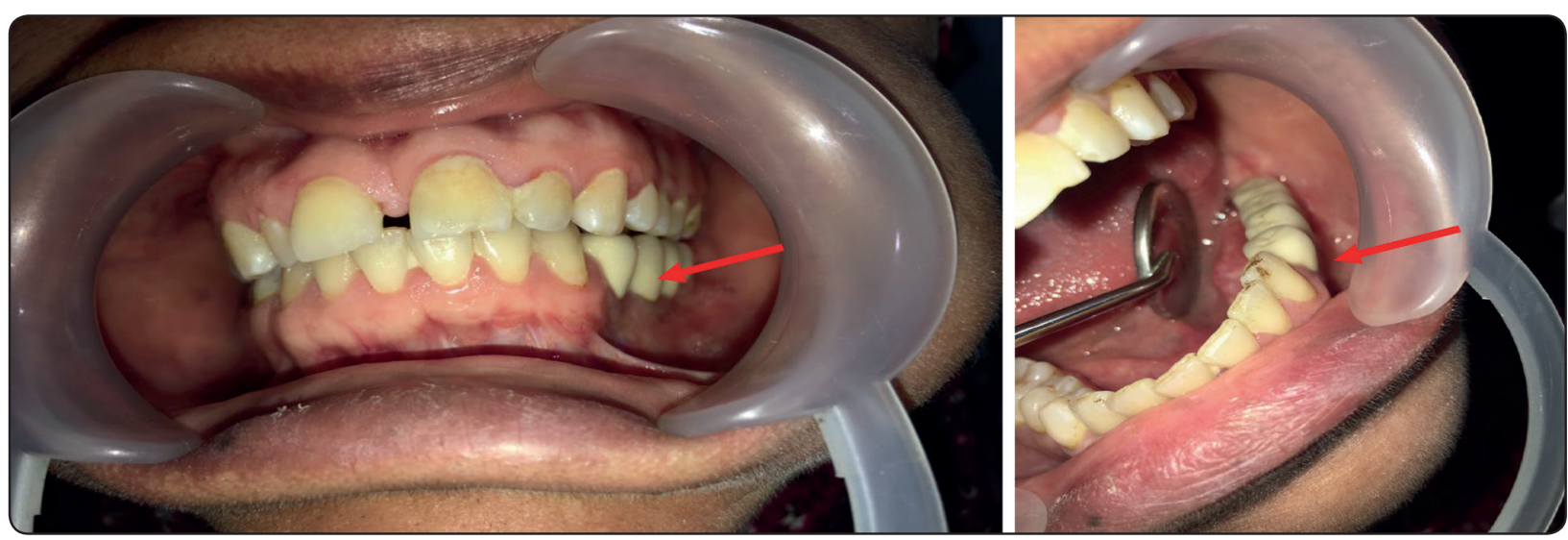

Fig. (8): Photograph showing intraoral total implant supported PFM prosthesis cementation

\section{[1] Clinical evaluation}

By recording the patient's satisfaction with regard to esthetic aspect of facial contour after tumor ablation and both esthetic and function results to the inserted implants supported prothesis according to a questionnaire with using a score on a (0-2) scale:

$$
\begin{aligned}
& 0=\text { not satisfied } . \\
& 1=\text { partially satisfied } . \\
& 2=\text { fully satisfied } .
\end{aligned}
$$

\section{[2] Radiographic evaluation}

By use of orthopantogramic and CBCT views to evaluate the marginal bone loss at the following times immediate after fixture installation, then at the time of final abutment fixation (6 months), finally after prosthetic loading (4 months). (Fig.9)

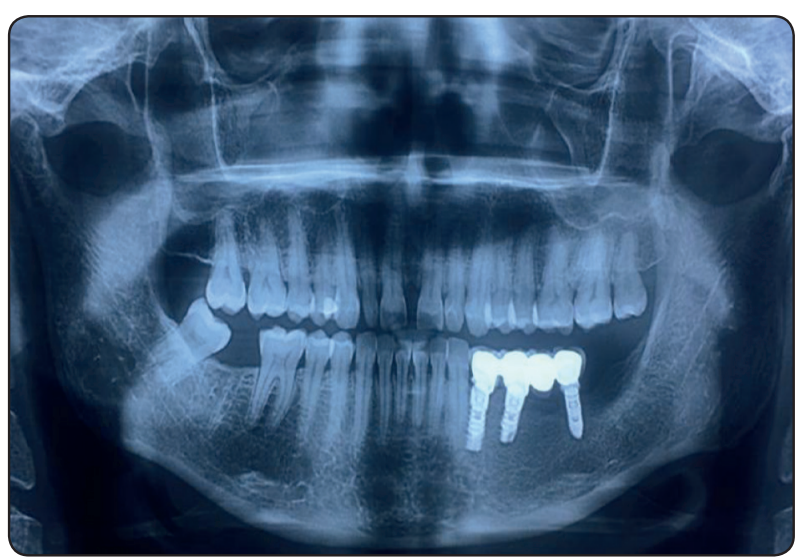

Fig. (9): Panoramic photoradiograph showing final prosthesis in occlusionPFM prosthesis cementation

\section{RESULTS}

The mean length of stay in the hospital in all patients was 1.5 days. Postoperative recovery after tumor ablatiom was uneventful in all the operated eight patients, with excellent native bone formation, as evidenced from the performed orthopantogramic views after 6 months from surgery. No dehiscence of the flap occurred. No infection had been detected in all the operated patients.

The postoperative recovery after insertion of the dental implants in the native bone (32 implants) was uneventful in 7 out of 8 patients. As, in patient No. (3) the four implants failed to integrate properly with bone before prosthetic loading and they were removed and waited for 6 months till the optimal bone regeneration occurred, then prepared for insertion of root form fixtures.

\section{Presence of pain around the inserted fixtures}

No pain had been detected in all the operated cases around the inserted implants, according to the results of (VAS) through the follow up periods except only one case (case no 3) suffered from pain through all the follow up periods (immediate and six months).

\section{Presence of peri-implant infection}

All patients healed properly as shown through the follow up periods. Only one patient (case no. 
3) showed presence of infection around the inserted fixtures with occurance of suppuration after three months from surgery and the suppuration was decreased, but not disappeared after injection of I.V. antibiotic (Cefotax $1 \mathrm{gm} / 12$ hours for 5 days), as evidence in the late follow up period (i.e. after six months from installation of the fixture).

\section{Presence of dental implant mobility:}

No dental implant mobility had been detected in all the operated cases immediately after insertion of dental implants. But in the late follow up periods (i.e. after six months) only one case (case no. 3) showed occurrence of mobility of the inserted four fixtures

\section{Presence of continuous peri-implant radiolucency}

Orthopantogramic and CBCT Views postoperatively showed occurrence of proper bone healing and integration around the inserted dental implants and no peri-implant radiolucency had been detected after six months from insertion of the fixtures in all cases, except in case no. (3), periimplant radiolucency had been detected through the late follow up period (i.e. 6 months), around the inserted four fixtures.

\section{The degree of bone resorption around the inserted dental implants}

Orthopantogramic and CBCT Views postoperatively showed proper formation of bone around the inserted fixtures in all cases through the follow up periods i.e. (6 and 10 months of insertion of the fixtures), except in case no. (3), where bone resorption occurred around the inserted fixtures after six months and ten months as evidenced by the following measurements.

No peri-implant radiolucency nor bone resorption had been detected in all cases after performing prosthetic rehabilitation, except in case no. (3), that showed occurrence of persistent peri-implant radiolucency and the mean of bone resorption was
(5) $\mathrm{mm}$ in all inserted four fixtures, after six months from fixture installation.

The results of peri-implant bone resorption through the follow up periods are reported in table (3), as follow:

TABLE (3): Showing Mean, Median and ranges of the peri-implant bone resorption

\begin{tabular}{|c|c|c|}
\hline $\begin{array}{c}\text { Peri-implant bone } \\
\text { resorption }\end{array}$ & $\begin{array}{c}\text { Six months } \\
\text { (abutment } \\
\text { installation) }\end{array}$ & $\begin{array}{c}\text { Ten months } \\
\text { (post prosthetic } \\
\text { loading) }\end{array}$ \\
\hline Mean & -0.67 & -0.80 \\
\hline SD & 0.156 & 0.119 \\
\hline Range minimum & -0.45 & -0.57 \\
\hline Maximum & -0.88 & -0.95 \\
\hline
\end{tabular}

\section{Patient satisfaction:}

All patients were fully satisfied with their esthetics, as good facial contour was obtained but seven out of eight patients were fully satisfied as far as the esthetic and functional aspects of their prosthetic restoration was considered, as one patient (case no. (3) lost all the four inserted dental fixtures. The satisfaction scores were reported in table (4).

TABLE (4): Showing score of patient's satisfactions

\begin{tabular}{|c|c|c|c|}
\hline $\begin{array}{c}\text { Patient } \\
\text { No }\end{array}$ & $\begin{array}{c}\text { Facial } \\
\text { contour }\end{array}$ & $\begin{array}{c}\text { Prosthesis } \\
\text { esthetics }\end{array}$ & $\begin{array}{c}\text { Prosthesis } \\
\text { function }\end{array}$ \\
\hline 1 & 2 & 2 & 2 \\
\hline 2 & 2 & 2 & 2 \\
\hline 3 & 2 & 0 & 0 \\
\hline 4 & 2 & 2 & 2 \\
\hline 5 & 2 & 2 & 2 \\
\hline 6 & 2 & 2 & 2 \\
\hline 7 & 2 & 2 & 2 \\
\hline 8 & 2 & 2 & 2 \\
\hline
\end{tabular}




\section{The success rate for the inserted implants}

The cumulative success rate for the inserted dental implants was $87.5 \%$ as 28 fixtures were succeeded to integrate from total of 32 fixtures.

\section{DISCUSSION}

The mean hospitalization of stage of tumor or cystic ablation was 1.5 days. This agrees with Matteo et. al., (2008), who reported that, the mean of hospitalization for tumor removal was 2 to 3 days. ${ }^{(1)}$

Tumor and cysts in oral and maxillofacial region can affects both soft and hard tissues. And any surgical interference such as tumor ablation or cyst enucleation leads to occurrence of residual defects in the native bone. However, these defects can be filled spontaneously with bone within time. In this study orthopantogramic views (6 months after surgery) showed occurrence of uneventful native bone formation in the resultant defects after ablation of the lesion. This result accepts with $\boldsymbol{D r}$ Richi Burman et. al., (2013)., who reported that, the resultant defects after ablation of tumors and cystic enucleation can be spontaneously filled with bone over a period of time. ${ }^{(9)}$

Performing oral rehabilitation by insertion of dental implants in the regenerated bone improve both function and aesthetics. This result agrees with Raghobar et al., (2008), who reported that, insertion of the dental implants in the regenerated bone can provide proper retention to the overlying prosthesis. ${ }^{(10)}$

In this study, no pain had been detected at the end of follow up period according to the VAS except in only one case, case no., (3) who showed presence of pain through all follow up periods. This agrees with Dr Richi Burman et.al.,(2013)., who reported presence of pain in the dental implants, that inserted in poor quality of regenerated bone. ${ }^{(9)}$

In the present study, only one patient should occurrence of mobility in all the inserted implants (four fixtures) at the end of follow up. This is in acceptance Matteo et. al., (2008), who reported occurrence of failure of the two inserted implants in only one case from twelve cases. ${ }^{(1)}$

According to the results of this study, no continuous peri-implant radiolucency had been detected in all cases except in only one case, case no., (3), this denotes to occurrence of proper bone healing and integration around the inserted dental implants in seven out of eight patients. This result agrees with Komisar (1990), who reported occurrence pf proper osseointegration of the inserted implants in the native bone after tumor ablation. ${ }^{(11)}$

In the present study, only one patient case no., (3) shoed presence of peri-implant infection at the late follow up (i.e. six months). This result agrees with Revista Cubama (2019), who reported that there were no signs of peri-implant and/or periodontal diseases in $95 \%$ of the performed cases. ${ }^{(12)}$

In this study, no bone resorption occurred around the inserted fixtures, as evidence by orthopantogramic and CBCT examination in all cases except in only one case, (case no. 3), where bone resorption occurred around all of the inserted fixtures and these four fixtures removed. This result agrees with Matteo Chiapasco et. al., (2008), who reported occurrence of very limited bone resorption rate of the native bone which occurs due to the prosthetic load which stimulate occurrence of functional stimulation to new bone formation. ${ }^{(1)}$

According to the results of this study, all patients were fully satisfied with esthetics, as good facial contour was obtained. But according to aesthetic and functional aspects of their prosthetic restoration, only one patient (case no. 3) was unsatisfied as loss of all of the four inserted fixtures occurred. This result is also consistent with those reported by Young et al., (2007). ${ }^{(13)}$

At the end of this study, the success rate for the inserted dental implants was excellent in all treated 
cases, except (case no. 3). This result agrees with Abdulwassie et al., (2008), who reported that, implants placed in the regenerated bone shows the same clinical, radiological and histomorphometric characteristics as those placed in the normal bone. ${ }^{(14)}$

\section{CONCLUSION}

The obtained results from this study demonstrated that, proper bone healing occurred after ablation of cystic or benign tumor lesions. It has also been shown that, the patient satisfaction both functionally and aesthetically and the success rates of the implants inserted in the regenerated bone may guarantee on excellent prognosis of the implant supported prothesis.

\section{REFERENCES}

1. Matteo Chiapasco, Giacomo Colletti, EugenioRomeo, Zaniboni and Roberto Brusati, (2008): Long Term results of mandibular reconstruction with autogenous bone grafts and oral implants after tumor resection, clinical oral implant research 19, 1074-1080.

2. Matteo Chiapasco, Giacomo Colletti, EugenioRomeo, Zaniboni and Roberto Brusati, (2006): Clinical outcome of dental implants placed in fibula-free flaps used for the reconstruction of maxillomandibular defects following ablation for tumors or osteoradionecrosis. clinical oral implant research 17, 220-228.

3. Urken ML, Moscoso JF and Lawson (1994): Systemic approach to functional reconstruction of the oral cavity following partial and total glossectomy. Arch otolaryngal head neck surgery.120:589.

4. Zlotolow IM, Huryn JM and Piro JD(1992): Osseointegrated implants and functional prosthetic rehabilitation in microvascular fibula free flap reconstructed mandibles. Am.J.Surg.164:677.
5. Riediger D., (1988): Restoration of masticatory function by microsurgically revascularized iliac crest bone grafts using endosseous implants. J. plastic reconstructive surgery. 81:861.

6. Parel SM., Branemark PI and Jansson T. (1986): Osseointegration in maxillofacial prosthetics. Part I: International application. J. Prosthetic Dent.55:490.

7. Ecklet U, Schemider M, Erasmus F, Gerlach K, Kuhlisch E, Loukota R, Schubert J and Terheyden H, (2006): Open versus closed treatment of fractures of the mandibular condylar process, a prospective randomized multicenter study J., craniomaxillofacial surgery.34:306-314 .

8. Valaderma P, Oates TW, Jones AA, Simpsom J, Schoolfield JD and Cochran DL(2017) Evaluation of two different resonance frequency devices to detect implantstability: aclinical trial . J Periodontal.78:262-272.

9. Dr Richi Burman, Dr MD Rabivl Islam and Dr Anuj Sharma (2013): Evaluation of osseointegrated implants in regenerated Jaw Bones after cyst enculation. IOSR Journal of Dental and Medical sciences (IOSR-JDMS) volume 7, Issue 4, pp, 01-06.

10. Raghobar GM, MeiJer HJ, Stegenga B, VantsHof MA, Vanoort RP and Vissin KA (2008): Effectiveness of three treatment modalities for the edentulous mandible. A five year eandomized clinical trial. J clinical Oral implant Research. 11:195-201.

11. Komisar A. (1990): The functional result of mandibular reconstruction. J. Laryngoscopy. 100:364.

12. Revista Cubana de Estomatologia (2019): Oral Rehabilitation after removal of a central cemento-ossifying fibroma; 56(2):e 1985.

13. Young C.W., PogrelM.A. and Schmidt B.L. (2007): Quality of life in patients undergoing mandibular resection and staged reconstruction. Journal Oral \& Maxillofacial Surgery.65:706-712.

14. Abdulwassie, Hassan and Dhanrajani D.J. (2008): Prosthodontic implant rehabilitation after the treatment of a pathologic lesion in the mandible: a case report. Implant Dentistry, 10(3)178-181. 